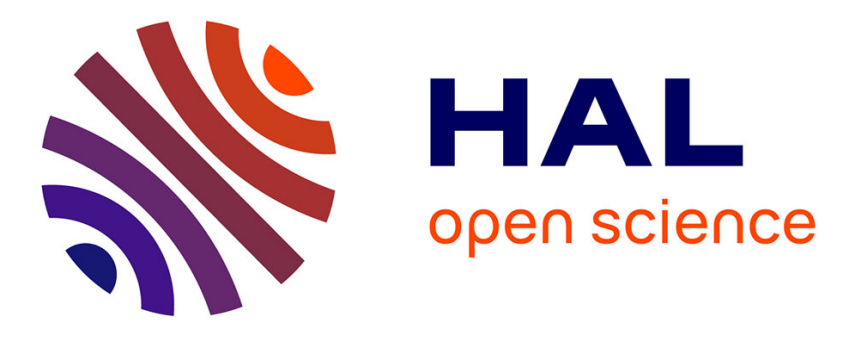

\title{
3D printing for cyclonic spray chambers in ICP spectrometry
}

Valérie Geertsen, Elodie Barruet, Olivier Tache

\section{To cite this version:}

Valérie Geertsen, Elodie Barruet, Olivier Tache. 3D printing for cyclonic spray chambers in ICP spectrometry. Journal of Analytical Atomic Spectrometry, 2015, 30 (6), pp.1369-1376. 10.1039/C5JA00045A . hal-01187815

\section{HAL Id: hal-01187815 https://hal.science/hal-01187815}

Submitted on 17 Nov 2015

HAL is a multi-disciplinary open access archive for the deposit and dissemination of scientific research documents, whether they are published or not. The documents may come from teaching and research institutions in France or abroad, or from public or private research centers.
L'archive ouverte pluridisciplinaire HAL, est destinée au dépôt et à la diffusion de documents scientifiques de niveau recherche, publiés ou non, émanant des établissements d'enseignement et de recherche français ou étrangers, des laboratoires publics ou privés. 


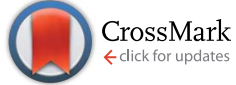

Cite this: J. Anal. At. Spectrom., 2015 30, 1369

Received 9th February 2015

Accepted 27th March 2015

DOI: 10.1039/c5ja00045a

www.rsc.org/jaas

\section{D printing for cyclonic spray chambers in ICP spectrometry $\dagger$}

\begin{abstract}
Valérie Geertsen,* Elodie Barruet and Olivier Taché
Additive manufacturing (AM) or 3D-printing is an increasingly widespread technique which is often described as a source for rapid prototyping whereas it is a manufacturing process in itself. It is a new tool for instrumental research laboratories which can now easily manufacture by themselves a large variety of devices. This article describes its application to ICP introduction system spray chambers. We undertake to print and study cyclone spray chambers by combining and comparing for the first time 3 different AM processes, 5 materials and 8 designs. The analytical performances of these spray chambers are compared with commercial glass and PFA chambers in terms of signal intensity, stability, oxide ratio, LOD and washout time. LODs measured with polymer printed chambers are in the range or even outperform those measured with the glass chamber even though 3D-printed chambers provide lower results in terms of sensitivity than glass. Compared to PFA chambers, the printed chambers are superior in terms of LOD. At low temperature, the printed chambers' efficiency depends on both AM process and manufacturing material. SLA and FDM printers give lower results in terms of sensitivity but not in LOD than the Polyjet printer. This study also illustrates the influence of the inner shape of the side arm nebulizer and confirms the importance of a free aerosol recirculation current around the nebulizer tip. Transfer tube efficiency is also questioned; it is found to be weakly detrimental to the sensitivity to light elements but shows no influence on heavy ones as well as on the stability or oxide ratio, whatever the element.
\end{abstract}

\section{Introduction}

In 2012, a normalization organisation, The American Society for Testing and Material (ASTM), defined additive manufacturing (AM) as a "process of joining materials to make objects from 3D model data, usually layer upon layer, as opposed to subtractive manufacturing methodologies, such as traditional machining”. Seven AM processes are listed by the ASTM: vat photo-polymerization, material jetting, binder jetting, material extrusion, powder bed fusion, sheet lamination and direct energy deposition. These processes differ not only in the size of the object they manufacture but also in the material they print and the surface roughness they can achieve. "Additive manufacturing" is a term generally applied to describe the technology overall and more specifically industrial applications and professional high-end equipment and applications. Numerous alternative terms to "additive manufacturing" can be found such as "3D printing”, "E-manufacturing”, "freeform fabrication”, .... These terms were coined after "rapid prototyping" which described in a very limited manner the process possibilities and applications. 3D printing is not only a process for rapid prototyping but

Commissariat à l'Energie Atomique et aux Energies Alternatives, CEA Saclay, DSM/IRAMIS/NIMBE/LIONS, CNRS UMR 3685, 91191 Gif Sur Yvette, France. E-mail: valerie.geertsen@cea.fr

$\dagger$ Electronic supplementary information (ESI) available. See DOI: 10.1039/c5ja00045a also a manufacturing technique in itself allowing new designs such as light-weight hollow structures or customized objects. Numerous applications of AM are reported in the literature using commercial printers (e.g. microchip or reaction vessel printing) and with new printing technologies (additive nanomanufacturing for nanolithography, ...). ${ }^{\mathbf{1 , 2}}$

ICP spectrometry is a mature analytical technique which has evolved largely since its invention in 1980. This technique provides elemental or isotope measurements with good accuracy over a very large concentration range. Its analytical performances (sensitivity, stability, LOD, ...) are continuously improving, mainly because of the progress in the field of electronics. Numerous introduction systems are available. ${ }^{3}$ The most common one consists of a pneumatic nebulizer producing a primary aerosol from which coarser droplets are removed in a spray chamber. A tertiary aerosol is then obtained and introduced into the plasma. It is generally accepted that water droplets with diameters smaller than $8 \mu \mathrm{m}$ should be considered as being the only ones able to complete the sequential process of vaporization, atomization and ionization, and contribute to the signal. ${ }^{4}$ The aerosol proportion which is analyzed or at least introduced into the plasma is low, ${ }^{5,6}$ the maximum attainable analyte transport efficiency for classical spray chambers at a high liquid sample flow rate $\left(1 \mathrm{~mL} \mathrm{~min}{ }^{-1}\right)$ reaches a few percent. This proportion can be improved both by increasing the number of droplets produced by the nebulizer 
which are sufficiently small to be analyzed and by optimizing the nebulization chamber geometry which must not only remove coarser droplets but also transport efficiently the smallest ones to the torch.

There are many selection criteria for a specific spray chamber, such as inner volume, $D_{3,2}$ cutoff diameter, memory effect, signal stability and intensity, oxide ratios, detection limit, equilibration time, transport efficiency, matrix effects, $\ldots .{ }^{7-9}$ Many chamber geometries have been reported in the literature. ${ }^{10}$ Double-pass spray chambers largely used in the past are today mainly replaced by single pass chambers or cyclones. The design of a cyclone itself presents numerous variants. Between 1980 and 1982, Greenfield, ${ }^{11}$ Thelin $^{12}$ and Ebdon ${ }^{13}$ compared double-pass Scott chambers with $750 \mathrm{~mL}$-volume conical cyclones. Cyclone spray chambers were found to transport a greater proportion of aerosol to the plasma than double-pass Scott chambers. The authors reported increases in signal to background ratios by a factor of 1.4 to 2.5. In 1986 A. Montaser et al. reported the study of two 220 and $60 \mathrm{~mL}$-cyclone chambers based on the basic configuration of the industrial tall and narrow cyclone. ${ }^{14}$ They showed that the signal to background ratios, detection limits and precisions of the analyte signal intensities obtained with the small cyclone chamber were slightly superior to those achieved with a Scott spray chamber or with a gravitational sedimentation chamber. ${ }^{15}$ These results were recently confirmed with the study of the commercial socalled Sturman-Master chamber. ${ }^{16-18}$ Hieftje and coworkers also proposed vertical cyclones, called rotary cyclones, combining gravitational, centrifugal, turbulent and impact aerosol-sizing mechanisms in a single apparatus. ${ }^{\mathbf{1 9 , 2 0}}$ Commercial vortex cyclonic chambers manufactured from various materials (polypropylene, PTFE and glass) were also compared with double-pass Scott chambers. ${ }^{\mathbf{8 1 0 , 2 1 , 2 2}}$ The authors showed that the position of the nebulizer inside the chamber had a noticeable effect on the performances. Cyclones can also include spoilers to deviate the aerosol flow inside the chamber, thus reducing wash-out time. ${ }^{23}$ A vertical aerosol transfer tube can be introduced inside the chamber to enhance the ICP signal and shorten both wash-out and equilibration times. ${ }^{3}$

In spite of the margin of improvement that can be expected from spray chamber optimization, this subject is rarely reported today. This is probably due to the need for glassblowing, an activity which is generally outsourced. Additive manufacturing then appears to be a perfect tool to manufacture customized objects in the laboratory itself. Also, the 3D-printing of spray chambers has recently been demonstrated with a low-cost home printer by D. F. Thompson. ${ }^{24}$

It is well known that the main phenomena and processes that have to be considered in the spray chamber are aerosol flow turbulence, gas phase compressibility, evaporation of droplets, as well as coalescence and impact of droplets which are based on gravitational settling or inertial deposition. ${ }^{25}$ For vortex cyclone chambers, the impact of droplets on the chamber inner surface is the main process involved in the elimination of the droplets as shown by Schaldach and coworkers. The fluid dynamics phenomena that occur when droplets impact solid or liquid surfaces are very complicated. They depend on many different parameters such as droplet liquid surface tension, viscosity, density and temperature, droplet diameter, impact angle, droplet velocity but also wall physical and chemical properties, surface roughness and temperature. ${ }^{26}$ An influence of the printing material and the fabrication process on spray chamber performances can thus be expected.

We undertake to print and study cyclone spray chambers by combining 3 different AM processes, 5 materials and 8 designs. The analytical performances of these spray chambers are compared with those of commercial glass and PFA chambers in terms of signal intensity, stability, oxide ratio, LOD and washout time. The first part of this work focuses on the influence of the printing process and the material, the second one on spray chamber geometry optimization via two distinct studies: the nebulizer side arm and the aerosol transfer tube.

\section{Materials and methods}

A 1 ppb multi-element solution is prepared by weight dilution in $2 \% \mathrm{HNO}_{3}$ from $1000 \mathrm{mg} \mathrm{L}^{-1}$ mono-elemental Li, Co, In, Ce, Ba, $\mathrm{Bi}$, and $\mathrm{U}$ standards (Spex-CertiPrep, Metuchen, USA). Nitric acid is volume-diluted from Merck 60\% ultrapure.

The quadrupole ICPMS is an iCAPQ (ThermoElectron) classically equipped with glass or PFA nebulization chambers cooled using a Peltier device. Glass and PFA commercial chambers are approximately $40 \mathrm{~mL}$ cyclones and include aerosol transfer tubes (Fig. 1). Comparison between spray chambers is performed after automatic tuning of the instrument which acts on the torch position, extraction and focus lenses, and nebulizer gas flow. The entire study is realized with a $1 \mathrm{~mL}$ $\min ^{-1}$ concentric glass nebulizer (Conikal, Glass Expansion) with natural uptake. The glass spray chamber is regularly tested to confirm the stable day-to-day performance of the ICPMS. The operating conditions are listed in Table 1.

All measurements (blank and samples) were performed on 6 replicates. Each replicate was a 10-run average measurement. The dwell time was fixed at $0.04 \mathrm{~s}$, there were 5 channels per mass, spaced at 0.01 amu. The LOD was calculated according to

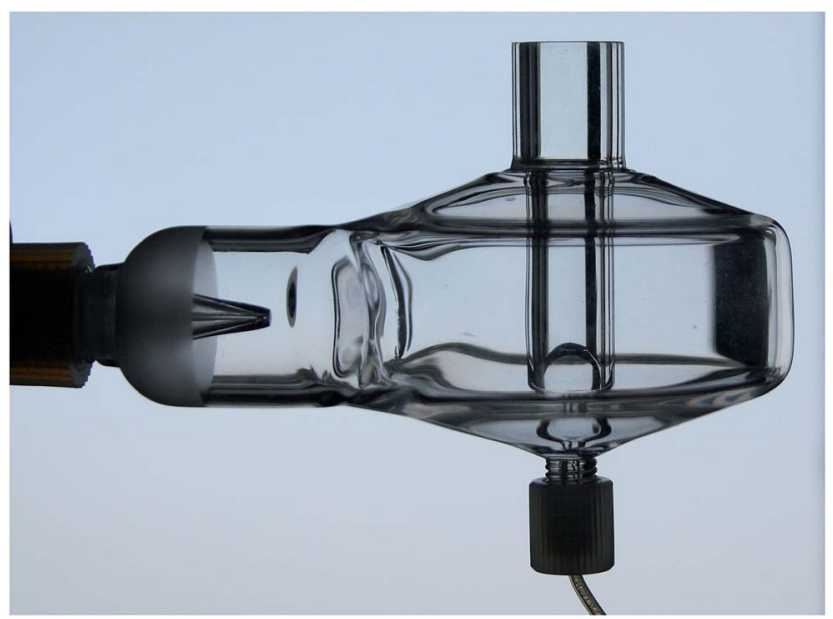

Fig. 1 Side view of the glass spray chamber equipped with the nebulizer. 
Table 1 iCAP Q ICPMS operating conditions

\begin{tabular}{ll}
\hline Parameter & Value \\
\hline Generator forward power & $1550 \mathrm{~W}$ \\
Nebulizer gas flow range & $0.8-1.2 \mathrm{~L} \mathrm{~min}^{-1}$ \\
Plasma gas flow rate & $14 \mathrm{~L} \mathrm{~min}^{-1}$ \\
Auxiliary gas flow rate & $0.8 \mathrm{~L} \mathrm{~min}$ \\
Sample flow rate & Natural uptake
\end{tabular}

the $3 \sigma$ criterion, where $\sigma$ is the standard deviation of 6 blank replicates. The short-term stability (10 $\mathrm{min})$ was evaluated by calculating the RSD of 40 replicates.

The temperature measurements were realized with a thermocouple directly inserted into the spray chamber transfer tube.

Printed PLA surface modification was realized in a plasma cleaner (Harrick Plasma). The spray chamber was introduced in a reaction enclosure under low vacuum. Low flow rates of oxygen at low pressure were then introduced in the enclosure and subjected to radio frequency electromagnetic radiation creating a plasma at near ambient temperature. The plasma ionized gas molecules interacted with the PLA solid surfaces modifying their physical and chemical characteristics.

\section{Nebulization chamber description}

The first set of spray chambers comprises 6 chambers of identical shape (M) but differing materials and/or printing processes (Table 2). PLA M, PLAD M and PLAcarb M are made with lowcost consumer printers at medium resolution $(200 \mu \mathrm{m})$. PLA M and PLAD $M$ are both printed with high quality polylactic acid filaments (PLA) while PLACarb, a polymer with better layer adhesion than PLA, is made of polylactic acid and carbon fiber. PMMA M is printed by jetting through nozzles tiny droplets of a liquid or gel-like photopolymer which are instantly UV-cured. This technology provides high-definition printing with $28 \mu \mathrm{m}-$ layers. The polymethacrylate resin chamber (PMA M) is made by medium resolution stereolithography (SLA), an optical system that directs a laser across a tank of liquid resin, solidifying layers as the solid is drawn below the liquid surface. The layers reach a thickness of $100 \mu \mathrm{m}$. The PLA $\mathrm{O}_{2}$ is a PLA M chamber treated with cold low-pressure oxygen plasma to increase surface wettability.

The $\mathrm{M}$ geometry is illustrated in Fig. 2. It is very close to the glass chamber geometry from which it differs by the inner shape of the nebulizer's side arm. The glass spray chamber nebulizer

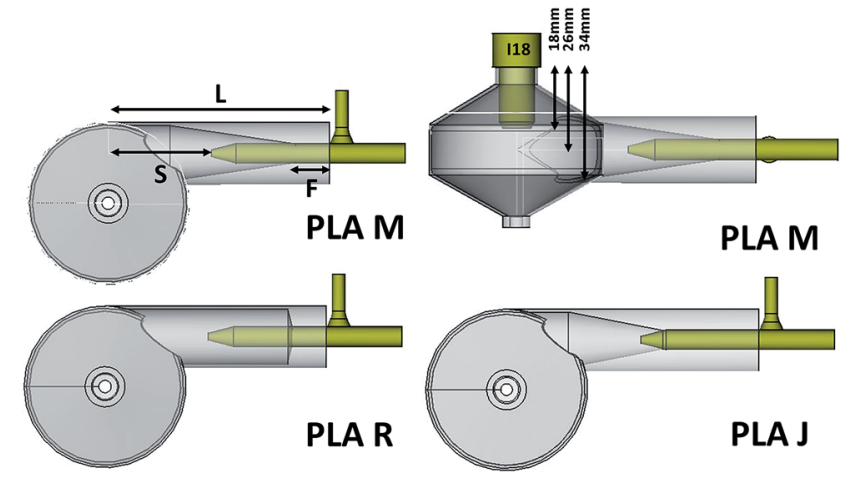

Fig. 2 Top and side views of various spray chambers with the nebulizer and transfer tube $\mathbf{I 1 8 .}$

arm is a simple tube attached to the cyclone body (Fig. 1). In the M shape, the nebulizer arm opening is conical for better evacuation of the very large droplets that often stagnate in a highflow nebulizer.

The second set of chambers is made of 7 PLA cyclones built identical to PLA M but with various side arm dimensions. The chambers are described in Table $3 . L$ is the total arm length and $F$ is the distance over which the nebulizer is fitted inside the arm (Fig. 2). $F$ varies from 11 to $33 \mathrm{~mm}$, given that the distance between the gas entry and the nebulizer nozzle is $37 \mathrm{~mm}$. An example of these chambers is shown in Fig. 2. All PLA chambers have a conical hollow geometry except PLA $R$ which has a cylindrical geometry.

The printed chambers were designed using FreeCAD, an open source parametric 3D CAD model software

Table 3 PLA chamber's nebulizer side arm geometry

\begin{tabular}{lll}
\hline Chamber & $L(\mathrm{~mm})$ & $F(\mathrm{~mm})$ \\
\hline PLA H & 51 & 11 \\
PLA G & 61 & 21 \\
PLA I & 71 & 31 \\
PLA L & 71 & 21 \\
PLA M & 71 & 11 \\
PLA J & 81 & 31 \\
PLA K & 91 & 31 \\
PLA R & 71 & 11 \\
Glass & 71 & 20 \\
PFA & 71 & 33
\end{tabular}

Table 2 Printed chambers with M geometry

\begin{tabular}{|c|c|c|c|c|}
\hline Chamber & Process & Printer technology & Printer (supplier) & Material (supplier) \\
\hline PLA M & Material extrusion & FDM & Replicator2 (Makerbot) & PLA (Makerbot) \\
\hline PLAD M & Material extrusion & FDM & Ultimaker2 (Ultimaker) & PLA (ColorFab) \\
\hline PMA M & Vat photo polymerization & SLA & Form1+ (FormLabs) & PMA resin (FormLabs) \\
\hline PLAcarb M & Material extrusion & FDM & Replicator2 & PLA carbon reinforced (Proto Pasta) \\
\hline PMMA M & Material jetting & Polyjet & Object30 (Statrasys) & PMMA resin (Statrasys) \\
\hline $\mathrm{PLA} \mathrm{O}_{2} \mathrm{M}$ & Material extrusion & FDM & Replicator2 & PLA (Makerbot) \\
\hline
\end{tabular}


(http:/www.freecadweb.org). The free CAD files created were exported to the printers in the *.stl file format. Stl (stereolithography) is a format that only describes the surface geometry of the three-dimensional object. The printer software afterwards slices structures and monitors the manufacturing process, each printer having its own slicer software. Whatever the printer used, spray chambers were built horizontally. External pillars were added to support the structure (cyclone bottom and nebulizer side arm) either manually or automatically by the printer's software.

All chambers incorporate central transfer tubes as do the reference glass and PFA spray chambers. In the case of the 2 commercial chambers, central transfer tubes are attached to the cyclone ceiling, where additive building is impossible without adding supporting pillars inside the cyclone itself. To circumvent this difficulty, transfer tubes were built separately and inserted into the chambers. This provides an opportunity to study the transfer tube geometry effect on the performances. A set of 6 transfer tubes of various lengths were printed in PLA using Replicator 2, one of the filament printers listed in Table 2. An example of the transfer tube I18 is shown in Fig. 2. They are denoted as I $x, x$ being their total length. The insert used in this study is I22 if not specified otherwise.

\section{Results and discussion}

\section{$M$ geometry}

Fig. 3 compares the entire set of $\mathbf{M}$ geometry printed chambers with the glass chamber at ambient temperature. This comparison consists in measuring the signal intensities of 4 elements $\left({ }^{7} \mathrm{Li}, \quad{ }^{59} \mathrm{Co},{ }^{115} \mathrm{In}\right.$, and $\left.{ }^{238} \mathrm{U}\right)$ as well as oxide ratios $\left({ }^{144} \mathrm{Ce}^{16} \mathrm{O} /{ }^{144} \mathrm{Ce}\right)$ at various nebulization gas flow rates. The comparison of spray chambers only in terms of signal intensities is reductive as it is easy to enhance signal intensities by increasing the nebulizer gas flow rate even if it is detrimental to oxide ratios. On the other hand, fixing the nebulization gas flow rate is not judicious as its tuning is poorly reproducible from day to day. Working at fixed oxide ratios is possible but in practice very difficult to realize. Finally, the nebulization gas flow value not being an analytical figure of merit in itself we propose more practically to plot the variation of signal intensities versus oxide ratios. Fig. 3 has thus been experimentally obtained by varying the nebulization gas flow rate between 0.9 and $1.4 \mathrm{~L} \mathrm{~min}^{-1}$ and measuring signal intensities and oxide ratios. For each gas flow rate, signal intensities are then reported versus oxide ratios. The figure shows a very strong correlation between cerium oxide ratios and signal intensities. It is almost impossible to tune the instrument while maintaining the oxide ratios below $2.4 \%$ without sacrificing sensitivity. The 5 printed chambers and the glass chamber show close performances despite very different fabrication processes. This result would seem to show that, contrary to what was expected, cyclone chambers which primarily act as impactors are not influenced by their constituent materials.

It is well known that temperature plays a major role in the sensitivity of the introduction system. Vaporizing sample aerosol by convective or infrared heating improves plasma temperature homogeneity and robustness by increasing the analyzable sample amount whereas cooling the spray chamber removes water vapor by condensation, concentrating samples and thus enhancing signal intensities. ${ }^{27}$ However it is impossible to heat printed chambers above the aerosol vaporization temperature because of the low fusion temperature of filaments or UV-resins. The only alternative is to cool the chambers. Fig. 4 shows a comparison of the spray chambers at the lowest temperature achievable with the ICPMS cooling device, that is $2{ }^{\circ} \mathrm{C}$. Fig. S1, $\dagger$ which provides a view of Fig. 4 restricted to oxide ratios from 1.5 to $2.5 \%$, is available in the ESI. $\uparrow$ The results are very different from those obtained previously. A signal intensity increase is globally observed, confirming the importance of water condensation. It is possible to tune the instrument in order to obtain both oxide ratios below $2.5 \%$ and satisfying signal
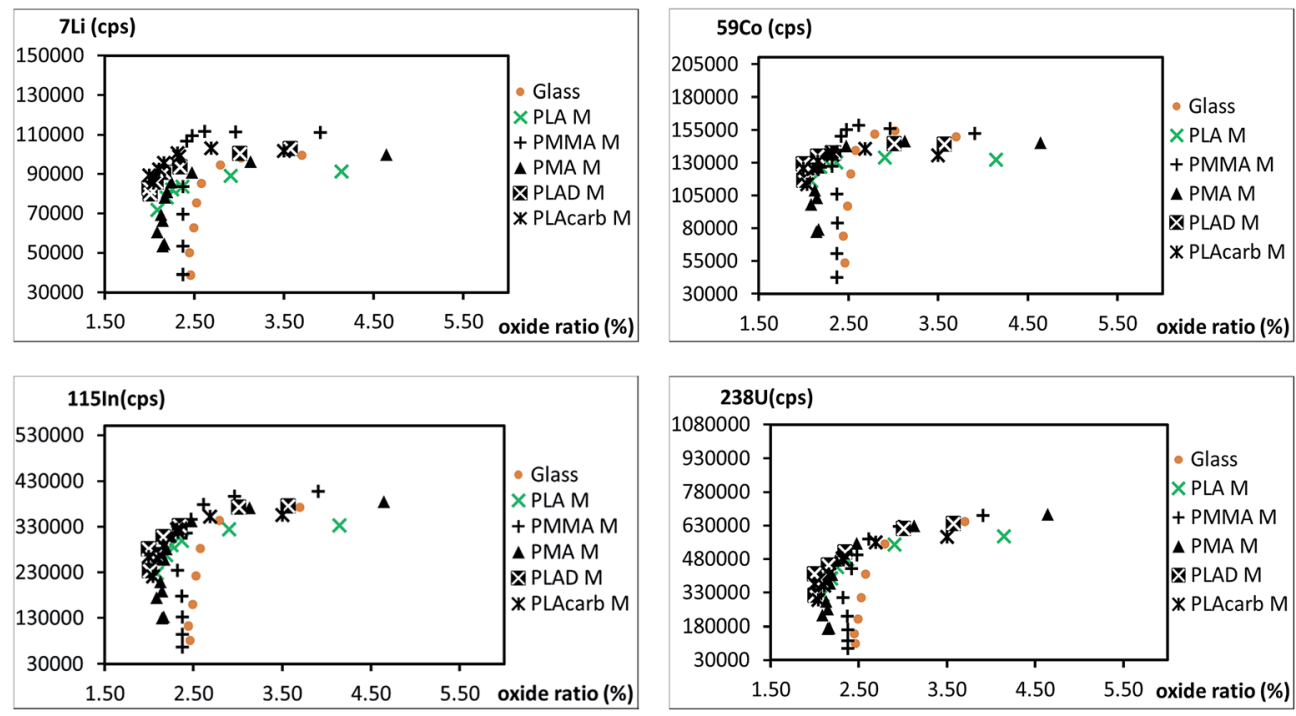

Fig. 3 Signal intensity versus cerium oxide ratios at ambient temperature for spray chambers with $\mathrm{M}$ geometry. 

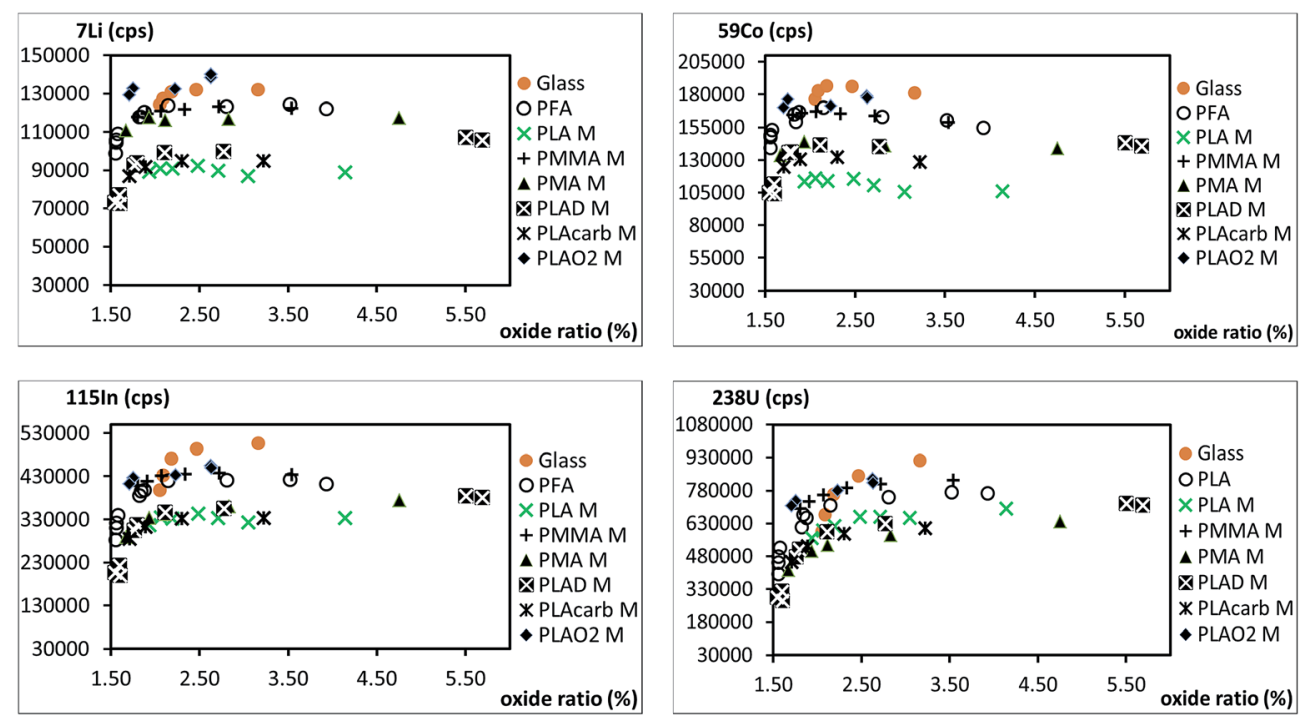

Fig. 4 Signal intensity versus cerium oxide ratios at low temperature for spray chambers with M geometry.

intensities. The polymer printed chambers are even capable of providing satisfying signals until the oxide ratio values reach as low as $1.7 \%$ which is impossible in the case of the glass chamber.

It can also be noted that the signal improvement is now material dependent. Glass shows the highest improvement, followed by the PMMA resin whereas the PMA resin and the three PLAs exhibit similar improvements. The similarity of the 3 PLA chamber performances indicates the absence of printer or filament influence. Two hypotheses are explored to explain the materials' different behavior towards temperature: the cooling could be more efficient inside the glass chambers compared to the plastic chambers enhancing water condensation or it could be a difference of the materials' hydrophilic properties.

The first assumption is based on glass and printing materials' thermal conductivity difference. Glass thermal conductivity is of the order of $1 \mathrm{~W} \mathrm{~m}^{-1} \mathrm{~K}^{-1}$ whereas the native thermal conductivity of polymers like PMMA or PLA is in the range of $0.2-0.4 \mathrm{~W} \mathrm{~m}^{-1} \mathrm{~K}^{-1}$. It could actually be even less due to the slight porosity introduced by the manufacturing process. A thermocouple is introduced inside the spray chamber transfer tubes at a fixed height to measure temperature versus Peltier cooling setting value. These measures show that the best temperature transfer is obtained on the PMMA chamber and not on the glass one. For a $4{ }^{\circ} \mathrm{C}$ goal temperature, the spray temperature is $8.4{ }^{\circ} \mathrm{C}$ in the PMMA chamber against $9.5^{\circ} \mathrm{C}$ for glass and $8.9{ }^{\circ} \mathrm{C}$ for PLA. This can be easily explained by the slight size difference between the printed and the glass chambers. The printed chambers fit perfectly the Peltier device for an optimized contact whereas the glass chamber being slightly smaller does not touch it on its entire external surface. These measures invalidate the temperature hypothesis as a source of signal variation. It is worth noting that the PLAcarb filament does not transfer heat better than the other PLAs despite the presence of $15 \%$ of carbon fiber. This is probably due to a circumferential alignment of the fiber that results from the printing process.
The second hypothesis is based on material wettability. An aqueous film is observed on the internal surface of the glass chamber when the droplets emitted by the nebulizer impact the surface or when the droplets condense on the cooled walls whereas printed chamber inner surfaces are covered with drops that magnify until they reach several millimeters and slide down from the wall. To confirm this hypothesis a second PLA M spray chamber is printed and introduced for a few minutes inside a low-pressure cold oxygen plasma enclosure. Cold plasma treatments are classically employed to temporarily modify surface properties thus increasing wettability or adhesion by adding highly reactive functions such as $\mathrm{OH}$ groups. ${ }^{28-30}$ The surface treatment realized here is probably of low efficiency as it is difficult in our configuration to create an efficient treatment inside the cyclone. To do so, the plasma must penetrate the cyclone through small openings (drain and transfer tube openings). It is very likely that the oxygen plasma had few contact with the inner walls. The oxygen plasma treated spray chamber is denoted as PLA $\mathrm{M} \mathrm{O}_{2}$ (Fig. 4). The comparison between PLA M and PLA $\mathrm{O}_{2} \mathrm{M}$ shows a signal improvement of the four elements studied. It reaches for example $100000 \mathrm{cps}$ for ${ }^{115} \mathrm{In}$. This improvement is attributed to the surface modification which can be visualized here as a decrease in the volume of the droplet attached to the inner surface chamber. It has to be noted that the importance of water affinity properties for a printed material and glass has been pointed out previously but the author proposed to increase polymer hydrophobicity. ${ }^{24}$ We propose here, in contrast, to increase the hydrophilic properties.

Another analytical figure of merit in comparing the introduction system is the signal stability. Whatever the printed spray chamber tested, the short-term stability evaluated by calculating the RSD from 40 replicates is better than $2 \%$. These results compare very well with the glass chamber showing that, under the conditions tested, the material has no influence on the signal stability. 
The wash-out time is also a feature that must be taken into account for evaluating spray chambers. It is calculated here as the time necessary to decrease the signal to $1 \%$ of its initial intensity. The results obtained show no material influence. An average value of $17 \mathrm{~s}$ is necessary to complete the wash out whatever the spray chamber.

The LOD is measured on 4 spray chambers at low temperature (glass, PFA, PLA M, PMMA M and PMA M) (Fig. 5). The printed chambers generally compare very well with and sometimes even outperform the glass chamber. Among the printed chambers, PMA M shows the best performances with better results than the glass chamber for all elements except barium. The poor results obtained for barium for all printed chambers can be explained by its probable presence at trace levels in the materials themselves. It is also interesting to note the low performances of the PFA chamber. It can be attributed to the low stability of the PFA chamber blank. The PFA blank stability ranges between 5 and $50 \%$ for the elements analyzed, whereas the other chambers' blank stabilities rarely exceed $5 \%$.

This first set of chambers was realized on 4 printers, 3 lowcost home printers (Replicator 2, Ultimaker 2, Form1+) and a professional one (Object30) in order to study the influence of printing quality and more specifically of surface roughness and construction material. An extrusion process as employed in FDM printers leads to ridged surfaces which are detrimental to liquid draining. Imperfections (unfused filament fragments) are also observed, especially on the internal upper surface. Material jetting and vat photo-polymerization produce smoother surfaces without being as smooth as glass or PFA. It is difficult to state which is the best printing solution (process and material) as each has specific strengths and weaknesses. However this study shows that 3D-printed chambers that can be produced in the laboratory in a few hours for less than $1 €$ of material even on low-cost printing devices produce better results in terms of sensitivity and LOD than costly commercial PFA ones. Looking more closely, it appears that the two FDM printers exhibit similar results whatever the analytical figure of merit studied. There is no influence here of the filament or the printer. The SLA printed chamber PMA M shows poor results in terms of signal intensity but yields the best results in terms of LOD. If we compare high definition professional and low-cost printers, it appears that the 3 low cost printers give lower results in terms of sensitivity but not in LOD than the high definition professional printer. It is, at this point of the study, impossible to interpret this observation in terms of printing as the limiting

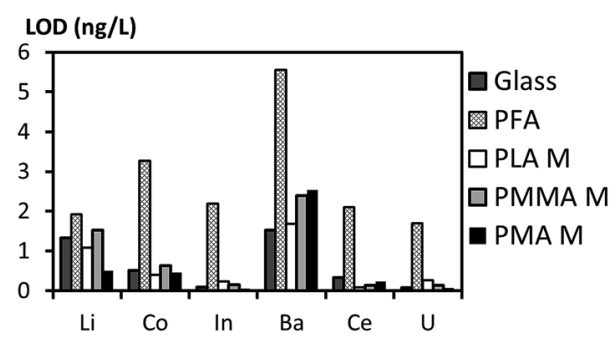

Fig. 5 LOD for several spray chambers at low temperature. factor here is the polymer wettability. Polymer hydrophilic properties must be improved before it is possible to comment on the influence of porosity or surface roughness.

\section{Nebulizer arm dimension}

The position of the nebulizer tip relative to the nebulization chamber and more precisely to the inner wall of the chamber has proved to be a parameter of great influence on the signal intensity. ${ }^{21,31}$ This figure of merit is classically evaluated by moving the nebulizer along the cyclone side arm. Additive manufacturing allows here printing of several chambers. It is then possible to study the effect on the signal of not only the total arm length $L$ but also of the tip's immediate surroundings. The comparison between PLA M and PLA J illustrates this last point, that is the chamber shape close to the nebulizer tip (Fig. 2). When $F$ is small, the nebulizer is held at a short distance, the tip is unobstructed allowing free circulation of gas flow streamlines. It also diminishes the evacuation slope of the deposited droplets allowing the formation of larger stagnating droplets under the tip. In contrast a large $F$ value enhances droplets draining, an important feature for chambers made of a hydrophobic material. Fig. 6 reports the influence of the cyclone side arm geometry on both ${ }^{115}$ In signal intensity and ${ }^{140}$ Ce oxide ratio. $\mathrm{H}$ and $\mathrm{K}$ geometries appear to be highly detrimental to the signal intensity. They coincide with the two extreme values of $L$ that is $51 \mathrm{~mm}$ and $91 \mathrm{~mm}$ giving distances $S$ from the nebulizer tip to the impact surface of 37 for PLA H and $75 \mathrm{~mm}$ for PLA K. At $37 \mathrm{~mm}$ from the nebulizer tip, the proportion of droplets in their ballistic phase is probably too high, leading to higher aerosol deposit on the impact area. ${ }^{25,32}$ Conversely, a very long distance to the impact area entails significant reduction of droplet speed, decreasing chamber performances. PLA K can be compared with PLA I and PLA J, the three chambers having the same $F$ distance, that is $31 \mathrm{~mm}$ and varying arm lengths. I and J geometries show the same performances whereas as described above, the $\mathrm{K}$ geometry is clearly unfavorable. This result is in agreement with a previous study published by Todoli et al. in 2000 where the influence of the nebulizer position is studied for several cyclonic chambers. ${ }^{21}$ The authors show that, for a Cinnabar cyclone using an o-ring seal adaptor fastening the nebulizer on a long distance, signal intensity is independent of the nebulizer position in a range of at least $7 \mathrm{~mm}$. The effect of $F$ at a fixed arm length $L$ is illustrated by the comparison of $\mathrm{M}, \mathrm{L}$

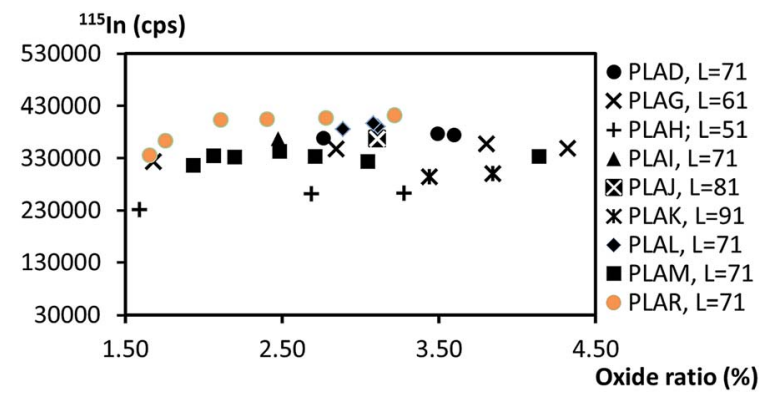

Fig. $6^{115}$ In signal intensity versus cerium oxide ratios at low temperature for spray chamber geometries described in Table 3. 
and I geometries. It can be seen that an optimal geometry is obtained at medium $F$, that is $21 \mathrm{~mm}$. This is in accordance with the observation of Sharp who recommends placing the nebulizer in the chamber so that the aerosol recirculation current comes from behind the nebulizer tip. ${ }^{32,33}$

It has to be noted that glass and PFA reference chamber geometries are slightly different from those of printed chambers. Glass and PFA chambers have large $F$ distances and flat surfaces under nebulizer tips even if this cylindrical hollow geometry is not efficient in terms of draining. PLA R, a new PLA chamber, is manufactured to study the inner shape influence (Fig. 2). PLA R is a PLA cyclone with a cylindrical inner shape of the side arm. It features the same $F$ and $L$ distances as PLA M and the same $F$ distance as both glass and PFA chambers. Fig. 6 shows that PLA R exhibits better sensitivity than PLA M and the best sensitivity of all printed chambers. This illustrates the improvement obtained by replacing a conical hollow side arm by a cylindrical one. This shows that the inner shape plays an important role in the aerosol distribution inside the cyclone and confirms the importance of the free aerosol recirculation current around the nebulizer tip. This subject should be studied precisely in the future to better understand the phenomenon and determine the optimal shape.

\section{Transfer tube}

Transfer tubes are often used in cyclonic chambers as a secondary particle separator to help remove larger aerosol particles. They are supposed to reduce solvent load in the plasma without compromising detection limits. Also, they are present in both glass and PFA commercial cyclones. To our knowledge the literature has never reported any study of their real impact. This can be easily explained by the difficulty and cost of performing such a study with glassware. In contrast this is very easy to realize with $3 \mathrm{D}$-printing. The length of the printed transfer tube varies here from $14 \mathrm{~mm}$ to $36 \mathrm{~mm}$. I26 is a transfer tube with an extremity in the nebulizer horizontal plane, I18 is entirely located in the upper part of the cyclone and I34 reaches the cyclone's conical lower part (Fig. 2). Transfer tubes are studied in terms of sensitivity and oxide ratio as described above. The results obtained for the 6 transfer tubes show decreasing impact with the element atomic weight. More precisely it has little influence on the sensitivity to light elements and no influence on heavy elements as well as on stability or

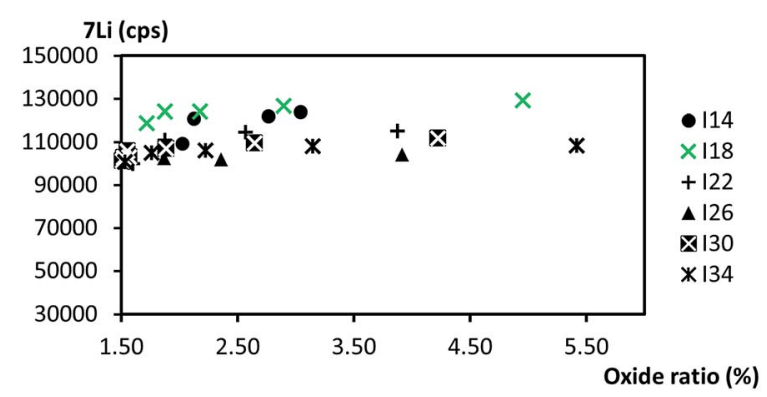

Fig. 7 Transfer tube influence on ${ }^{7} \mathrm{Li}$ signal intensity and cerium oxide ratio for the PMMA M spray chamber at low temperature. oxide ratio whatever the element. Fig. 7 illustrates the results obtained with the PMMA M chamber for the lightest element that is ${ }^{7} \mathrm{Li}$. I14 and I18 which are the shortest transfer tubes tested are the best in terms of sensitivity. These observations can be interpreted as a lack of transfer tube influence on heavy elements and a weak detrimental effect on light elements. This effect is especially disadvantageous as the tube transfer length in the cyclone increases and disturbs the aerosol circulation.

\section{Conclusion}

As demonstrated here, 3D printing is much more than a prototyping process; it is a new tool for scientists. The rapid turnaround of the process, the flexibility of design and the low cost of manufacturing provide scientists with previously non-existent possibilities for exploring geometries and materials.

This work demonstrates the applicability of AM for ICP spray chambers and is a basis for future studies. Multiple geometry adjustments of the chamber inner shape have been realized. They illustrate both the influence of the distance between the nebulizer tip and the impact area and the importance of aerosol circulation around the nebulizer nozzle. This last specific feature will be more largely developed in the future. These modifications will probably affect the outer chamber shape and may require chamber jacket cooling needing more advanced design and manufacturing.

Beyond the modification of the shape, 3D-printing with its wide range of printing materials provides a tool for studying the impact on chamber efficiency of other important parameters like the inner wall physical and chemical properties. The PLA polymer oxygen plasma treatment described here is the first example of the impact of the material wettability on signal intensity. 3D printing should contribute to an extensive study of surface treatment to improve sample washing, to reduce further LOD or to provide solutions to classical issues like boron memory effects. This may result in a variety of chambers with tailored surfaces for specific analytes competing with the existing commodity glass chambers.

\section{Acknowledgements}

The authors gratefully acknowledge Thomas Berthelot (CEA), Romain Di Vozzo (Digiteo FabLab) and Kevin Cedrone (FormLabs) for, respectively, the PMMA M, PLAD M and PMA M chamber printings. The authors also acknowledge the DIM Analytics of the Region Ile de France and the Plateau de Saclay RTRA program for the IcapQ funding.

\section{References}

1 A. M. Platform, Additive Manufacturing, Strategic Research Agenda, TCI, 2014.

2 M. D. Symes, P. J. Kitson, J. Yan, C. J. Richmond, G. J. T. Cooper, R. W. Bowman, T. Vilbrandt and L. Cronin, Nat. Chem., 2012, 4, 349-354, DOI: 10.1038/nchem.1313.

$3 \mathrm{~J}$. Todoli and J. Mermet, Liquid Sample Introduction in ICP Spectrometry: A Practical Guide. 2008. 
4 M. G. Minnich, J. A. McLean and A. Montaser, Spectrochim. Acta, Part B, 2001, 56, 1113-1126.

5 V. Geertsen, P. Lemaitre, M. Tabarant and F. Chartier, J. Anal. At. Spectrom., 2012, 27, 146-158, DOI: 10.1039/c1ja10255a.

6 D. S. Engstrom, B. Porter, M. Pacios and H. Bhaskaran, J. Mater. Res., 2014, 29, 1792-1816, DOI: 10.1557/jmr.2014.159.

7 N. H. Bings, J. O. O. von Niessen and J. N. Schaper, Spectrochim. Acta, Part B, 2014, 100, 14-37, DOI: 10.1016/ j.sab.2014.08.011.

8 J. L. Todoli and J. M. Mermet, Spectrochim. Acta, Part B, 2006, 61, 239-283, DOI: 10.1016/j.sab.2005.12.010.

9 B. L. Sharp, N. W. Barnett, J. C. Burridge and J. F. Tyson, J. Anal. At. Spectrom., 1987, 2, R167-R210, DOI: 10.1039/ ja987020167r.

10 S. Maestre, PhD Thesis, University of Alicante, 2002.

11 S. Greenfield and D. T. Burns, Anal. Chim. Acta, 1980, 113, 205-220, DOI: 10.1016/s0003-2670(01)93734-1.

12 B. Thelin, Analyst, 1981, 106, 54-59, DOI: 10.1039/ an9810600054.

13 L. Ebdon and M. R. Cave, Analyst, 1982, 107, 172-178, DOI: 10.1039/an9820700172.

14 P. A. Vieira, H. Zhizhuang, S. K. Chan and A. Montaser, Appl. Spectrosc., 1986, 40, 1141-1146, DOI: 10.1366/ 0003702864507512.

15 P. N. Keliher, D. J. Gerth, J. L. Snyder, H. Wang and S. F. Zhu, Anal. Chem., 1988, 60, R342-R368.

16 X. H. Zhang, H. F. Li and Y. F. Yang, Talanta, 1995, 42, 19591963, DOI: 10.1016/0039-9140(95)01675-9.

17 L. C. Trevizan, E. C. Vieira, A. R. A. Nogueira and J. A. Nobrega, Spectrochim. Acta, Part B, 2005, 60, 575-581, DOI: 10.1016/j.sab.2005.02.014.

18 A. Asfaw and G. Wibetoe, J. Anal. At. Spectrom., 2007, 22, 158163, DOI: 10.1039/b609531c.
19 M. Wu and G. M. Hieftje, Appl. Spectrosc., 1992, 46, 19121918, DOI: 10.1366/0003702924123647.

20 M. Wu, Y. Madrid, J. A. Auxier and G. M. Hieftje, Anal. Chim. Acta, 1994, 286, 155-167, DOI: 10.1016/0003-2670(94)801568.

21 J. L. Todoli, S. Maestre, J. Mora, A. Canals and V. Hernandis, Fresenius' J. Anal. Chem., 2000, 368, 773-779, DOI: 10.1007/ s002160000583.

22 J. L. Todoli, J. M. Mermet, A. Canals and V. Hernandis, J. Anal. At. Spectrom., 1998, 13, 55-62, DOI: 10.1039/a704342b.

23 G. Schaldach, H. Berndt and B. L. Sharp, J. Anal. At. Spectrom., 2003, 18, 742-750, DOI: 10.1039/b302052e.

24 D. F. Thompson, J. Anal. At. Spectrom., 2014, 29, 2262-2266.

25 G. Schaldach, L. Berger, I. Razilov and H. Berndt, J. Anal. At. Spectrom., 2002, 17, 334-344, DOI: 10.1039/b106024b.

26 A. Frohn and N. Roth, Dynamics of Drplets, Springler, Berlin, 2000.

27 Y. Makonnen, J. Burgener and D. Beauchemin, J. Anal. At. Spectrom., 2015, 30, 214-224, DOI: 10.1039/c4ja00258j.

28 Y. Zhao, A. Fina, A. Venturello and F. Geobaldo, Appl. Surf. Sci., 2013, 283, 181-187, DOI: 10.1016/j.apsusc.2013.06.078.

29 R. M. Rasal, A. V. Janorkar and D. E. Hirt, Prog. Polym. Sci., 2010, 35, 338-356, DOI: 10.1016/j.progpolymsci.2009.12.003.

30 L. Chaozong, N. Cui, N. M. D. Brown and B. J. Meenan, Surf. Coat. Technol., 2004, 185, 311-320, DOI: 10.1016/ j.surfcoat.2004.01.024.

31 J. L. Todoli and V. Hernandis, J. Anal. At. Spectrom., 1999, 14, 1289-1295.

32 B. L. Sharp, J. Anal. At. Spectrom., 1988, 3, 939-963, DOI: 10.1039/ja9880300939.

33 B. L. Sharp, J. Anal. At. Spectrom., 1988, 3, 613-652. 